\title{
Pulmonary metastasectomy in colorectal cancer: the PulMiCC trial
}

\author{
Tom Treasure, ${ }^{1}$ Lesley Fallowfield, ${ }^{2}$ Belinda Lees, ${ }^{3}$ Vern Farewell ${ }^{4}$
}

${ }^{1}$ Clinical Operational Research

Unit, Department of

Mathematics, University College

London, London, UK

${ }^{2}$ Cancer Research UK

Psychosocial Oncology, Brighton \& Sussex Medical School,

University of Sussex, Brighton, UK

${ }^{3}$ Clinical Trials and Evaluation Unit, Royal Brompton Hospital, London, UK

${ }^{4}$ MRC Biostatistics Unit, Institute of Public Health, University Forvie Site,

Cambridge, UK

\section{Correspondence to}

Tom Treasure, Clinica

Operational Research Unit, Department of Mathematics, University College London, 4 Taviton Street, London WC1H OBT, UK;

tom.treasure@gmail.com

Received 11 February 2011 Accepted 14 February 2011 Published Online First 10 May 2011

\section{ABSTRACT}

PulMiCC (Pulmonary Metastasectomy in Colorectal Cancer) is a randomised controlled trial funded by Cancer Research UK. Patients with a history of resected colorectal cancer who are found to have pulmonary metastases are first registered for evaluation and, if subsequently eligible for the trial, they are invited to be randomly allocated to 'active monitoring' or 'active monitoring with pulmonary metastasectomy'. The clinical outcomes are overall survival, relapse-free survival, lung function and patient-reported quality of life.

Pulmonary metastasectomy-that is, the surgical removal of nodules of metastatic cancer from the lungs - is very well established and widely accepted practice, most commonly for colorectal cancer. ${ }^{1}$ From that starting point, the Pulmonary Metastasectomy in Colorectal Cancer (PulMiCC) trial is best understood as an investigation to discover if active monitoring without pulmonary metastasectomy might result in survival similar to that reported in surgical series when like is compared with like. The evidence available for the effectiveness of metastasectomy is in the form of retrospective case reviews and observational studies. These have been collated in a formally conducted systematic review and a quantitative synthesis of data from 3504 patients in 51 studies. ${ }^{2}$ There were no randomised trials. The 5-year survival rates of patients who had pulmonary metastasectomy are of the order of $40 \%$. In only two reports is a denominator provided. These show that metastasectomy was performed in a selected $1-3 \%$ of patients with recurrent cancer. Thirty years ago the suggestion was made that this better than expected survival might be an effect of selection rather than surgery. ${ }^{3}$ Modelling studies using Thames Cancer Registry data pose the same question. ${ }^{4}$

The characteristics of patients in the many retrospective studies have been remarkably consistent since the 1960s: the patients are (in round figures) of average age 60 years, $60 \%$ male, $60 \%$ have a solitary metastasis, about $60 \%$ have low/ normal carcinoma embryonic antigen (CEA) and a median interval of about 36 months has elapsed since the primary resection. In multivariable analyses, the consistent features that favour longer survival are a single metastasis, a long interoperative interval ${ }^{5}$ and a low CEA. ${ }^{6}$ These three factors are clearly favourable features in terms of the aggressiveness of the cancer: they are prognostic features for all patients with colorectal cancer rather than being predictive of the surgical effectiveness of pulmonary metastasectomy.

However, practice has gone well outside the bounds set in the observational studies. In a recent survey among members of the European Society of Thoracic Surgeons (ESTS), multiple metastases were not a contraindication for $85 \%$ of responding surgeons, an interval of less than a year since the primary operation was acceptable for $64 \%$ and synchronous metastases for $50 \%{ }^{7}$ Whether the reported good survival rates are due to surgery or selection, it cannot be assumed that they will be replicated in a practice which has strayed well outside the criteria employed in the selection of the 3504 patients in the quantitative synthesis. In contrast to the stated practice of the members at large, the ESTS Working Group was unable to offer recommendations for pulmonary metastasectomy on the basis of its findings. ${ }^{8}$

The design of PulMiCC is founded upon an uncertainty that must exist in the advice given to many of these patients. Consider two scenarios where advice for or against metastasectomy is reasonably consistent. Patients who have a cluster of the favourable features, such as a single pulmonary metastasis which is evident only after several years and is growing slowly, might be offered metastasectomy as part of widely accepted current practice (although it should be noted that effectiveness of metastasectomy has not been shown and these patients are eligible for randomisation within PulMiCC). In contrast, patients with multiple bilateral metastases, evident within months and growing, are generally advised against metastasectomy. However, between these extremes are patients with a combination of some favourable and some unfavourable features; they are neither an easy 'yes' for metastasectomy nor a confident 'no'. If there is uncertainty, unbiased allocation is as good a basis as any other for making the decision. ${ }^{9} 10$

PulMiCC has a two-stage consent process (figure 1) derived from that used in the Mesothelioma and Radical Surgery (MARS) trial where it proved an effective strategy to recruit sufficient patients into a very challenging trial. ${ }^{11}$ In the first stage of PulMiCC, any patient with one or more pulmonary nodules which might be from colorectal cancer can be invited to enter the study with no commitment to randomisation. DVDs for medical staff and for patients have been prepared based on evidence-based best practice in presenting uncertainty to patients in a way that is open, even handed and, at the same time, supportive. ${ }^{12-14}$ The notion that haste or urgency are helpful should be 
Figure 1 Consent process for patients in the Pulmonary Metastasectomy in Colorectal Cancer (PulMiCC) trial. MDM, multidisciplinary meeting; MDT, multidisciplinary team.

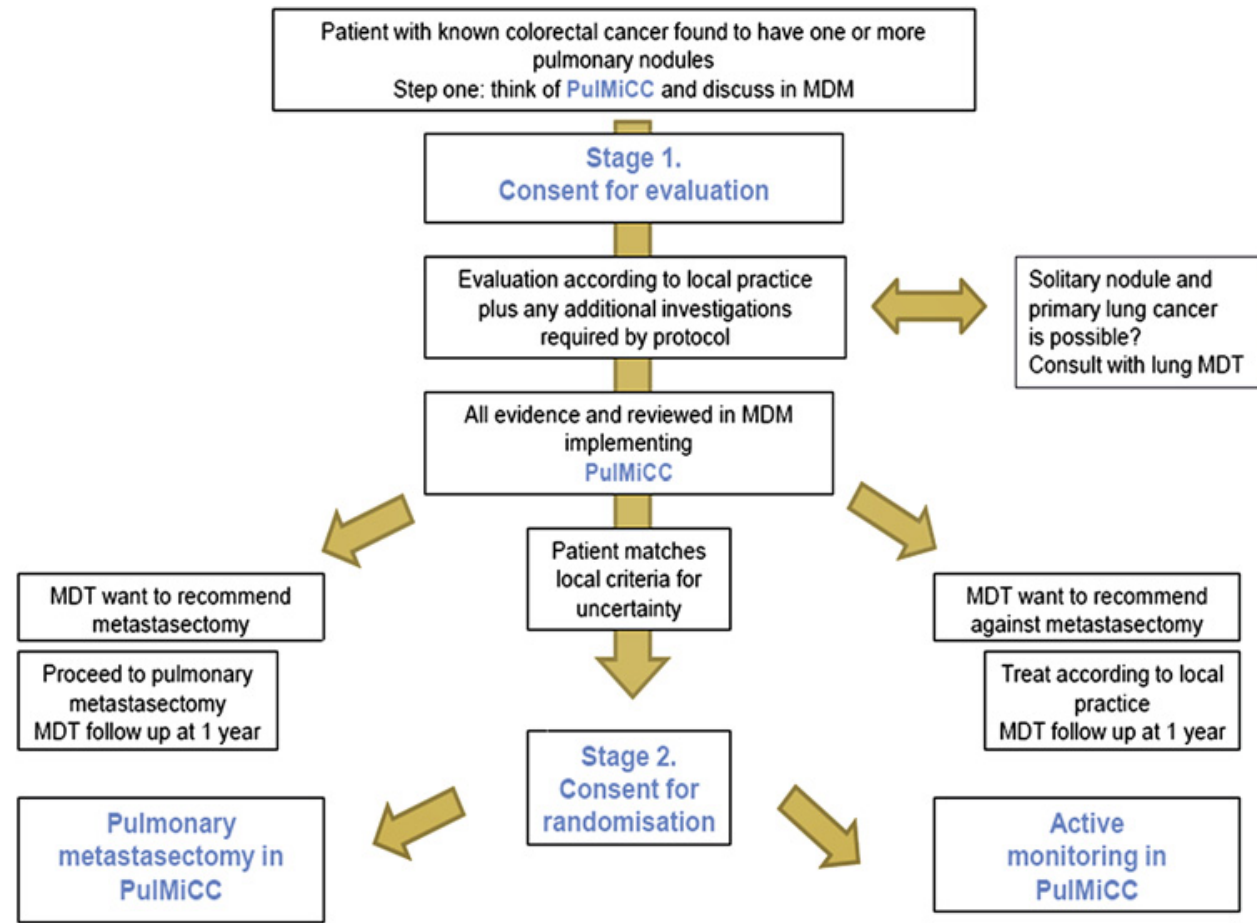

dispelled. ${ }^{15}$ Even if it is not easy to put these matters to patients, for those with demonstrable stage IV colorectal cancer, time measured in days or a couple of weeks is better spent in careful evaluation than hastening towards a thoracotomy.

During this first stage of PulMiCC the operative and pathological details of the primary cancer are reviewed. Where there is a solitary nodule, the possibility of primary lung cancer should be considered by the lung multidisciplinary team. If not already done, PET/CT is arranged. ${ }^{16}$ In some cases percutaneous biopsy will be advised. With regard to chemotherapy, we found no evident consensus. Some oncologists give chemotherapy routinely on discovery of recurrence, some believe it has a place in gauging the responsiveness of the disease and some use it as an adjuvant therapy. It can be given during this phase of the study. At the end of this phase there will be some patients for whom there is a decision against surgery and there will be some for whom metastasectomy will be offered on the basis of current practice and beliefs. Between these groups there is a zone of uncertainty.

For patients where there is uncertainty, a randomised allocation will be offered. The randomisation procedure will include minimisation to ensure that the two arms are similar in terms of age, sex, number of metastases, $\mathrm{T}$ and $\mathrm{N}$ stage of the primary, previous resection of liver metastases and CEA level. ${ }^{17}{ }^{18}$ Patients not allocated to metastasectomy will have "continued active monitoring'. Radiofrequency ablation or stereotactic radiotherapy are not treatment options within PulMiCC.

The surgical options are left to the local team to decide. The choice between thoracotomy and video-assisted thoracic surgery (VATS) is in large part governed by technical surgical considerations which depend on the size of the metastases and their exact location with respect to the pulmonary vessels. Some contend that the lung should be routinely palpated at thoracotomy to ensure that a complete metastasectomy is performed. ${ }^{19}$ This is a reminder that this surgery is not trivial, a notion which has become associated with metastasectomy in a VATS era. Data on symptomatic outcome are lacking and there are very few data on change in pulmonary function associated with metastasectomy. ${ }^{2}$ There must be some negative impact attributable to the chest wall incision, interference with the pleura and loss of lung parenchyma. These will be captured, along with patient-reported quality of life outcomes, pulmonary function and survival.

The trial is open for recruitment in an increasing number of UK centres. Full details of PulMiCC can be found on the website (http://www.rbht.nhs.uk/PulMiCC/).

Funding Cancer Reasearch UK.

Competing interests None.

Contributors $\Pi$ drafted the manuscript. All the authors have read and approved the submitted version.

Provenance and peer review Not commissioned; externally peer reviewed.

\section{REFERENCES}

1. Treasure T, Utley M, Hunt I. When professional opinion is not enough: surgical resection of pulmonary metastases. BMJ 2007:334:831-2.

2. Fiorentino F, Hunt I, Teoh K, et al. Pulmonary metastasectomy in colorectal cancer: a systematic review and quantitative synthesis. J R Soc Med 2010;103:60-6.

3. Aberg T, Malmberg KA, Nilsson B, et al. The effect of metastasectomy: fact or fiction? Ann Thorac Surg 1980;30:378-84.

4. Utley M, Treasure T. Interpreting data from surgical follow-up studies: the role of modeling. J Thorac Oncol 2010;5(6 Suppl 2):S200-2.

5. Onaitis MW, Petersen RP, Haney JC, et al. Prognostic factors for recurrence after pulmonary resection of colorectal cancer metastases. Ann Thorac Surg 2009;87:1684-8

6. Pfannschmidt J, Hoffmann H, Dienemann H. Reported outcome factors for pulmonary resection in metastatic colorectal cancer. J Thorac Oncol 2010;5 (6 Suppl 2):S172-8.

7. Internullo E, Cassivi SD, Van Raemdonck D, et al. Pulmonary metastasectomy: a survey of current practice amongst members of the European Society of Thoracic Surgeons. J Thorac Oncol 2008;3:1257-66.

8. Van Raemdonck D, Friedel G. The European Society of Thoracic Surgeons Lung Metastasectomy Project. J Thorac Oncol 2010;5(6 Suppl 2):S127-9.

9. Treasure T, Fallowfield L, Farewell V, et al. Pulmonary metastasectomy in colorectal cancer: time for a trial. Eur J Surg Oncol 2009:35:686-9.

10. Treasure T, Fallowfield L, Lees B. Pulmonary metastasectomy in colorectal cancer: the PulMiCC trial. J Thorac Oncol 2010;5(6 Suppl 2):S203-6.

11. Treasure $\mathbf{T}$, Waller $\mathrm{D}$, Tan $\mathrm{C}$, et al. The mesothelioma and radical surgery randomized controlled trial: the MARS feasibility study. J Thorac Oncol 2009:4:1254-8.

12. Jenkins V Vallowfield $L$. Reasons for accepting or declining to participate in randomized clinical trials for cancer therapy. Br J Cancer 2000;82:1783-8. 
13. Jenkins V, Fallowfield L, Saul J. Information needs of patients with cancer: results from a large study in UK cancer centres. Br J Cancer 2001;84:48-51.

14. Jenkins V, Fallowfield L, Solis-Trapala I, et al. Discussing randomised clinical trials of cancer therapy: evaluation of a Cancer Research UK training programme. BMJ 2005;330:400

15. Myrdal G, Lambe M, Hillerdal G, et al. Effect of delays on prognosis in patients with non-small cell lung cancer. Thorax 2004;59:45-9.
16. Detterbeck FC, Grodzki T, Gleeson $\mathrm{F}$, et al. Imaging requirements in the practice of pulmonary metastasectomy. J Thorac Oncol 2010;5(6 Suppl 2):S134-9.

17. Treasure $\mathbf{T}$, MacRae KD. Minimisation: the platinum standard for trials? BMJ 1998:317:362-3.

18. Altman DG, Bland JM. Treatment allocation by minimisation. BMJ 2005;330:843

19. Molnar TF, Gebitekin C, Turna A. What are the considerations in the surgical approach in pulmonary metastasectomy? J Thorac Oncol 2010;5(6 Suppl 2):S140-4.

\section{Images in Thorax}

\section{Pulmonary hamartoma mimicking primary bronchoalveolar cell}

\section{carcinoma}

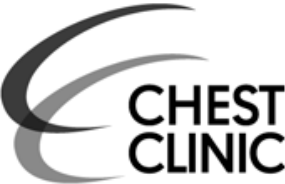

A 52-year-old man with transitional cell CLINIC

carcinoma underwent a chest CT because of a newly developed pulmonary nodule observed on a chest radiograph. In addi-
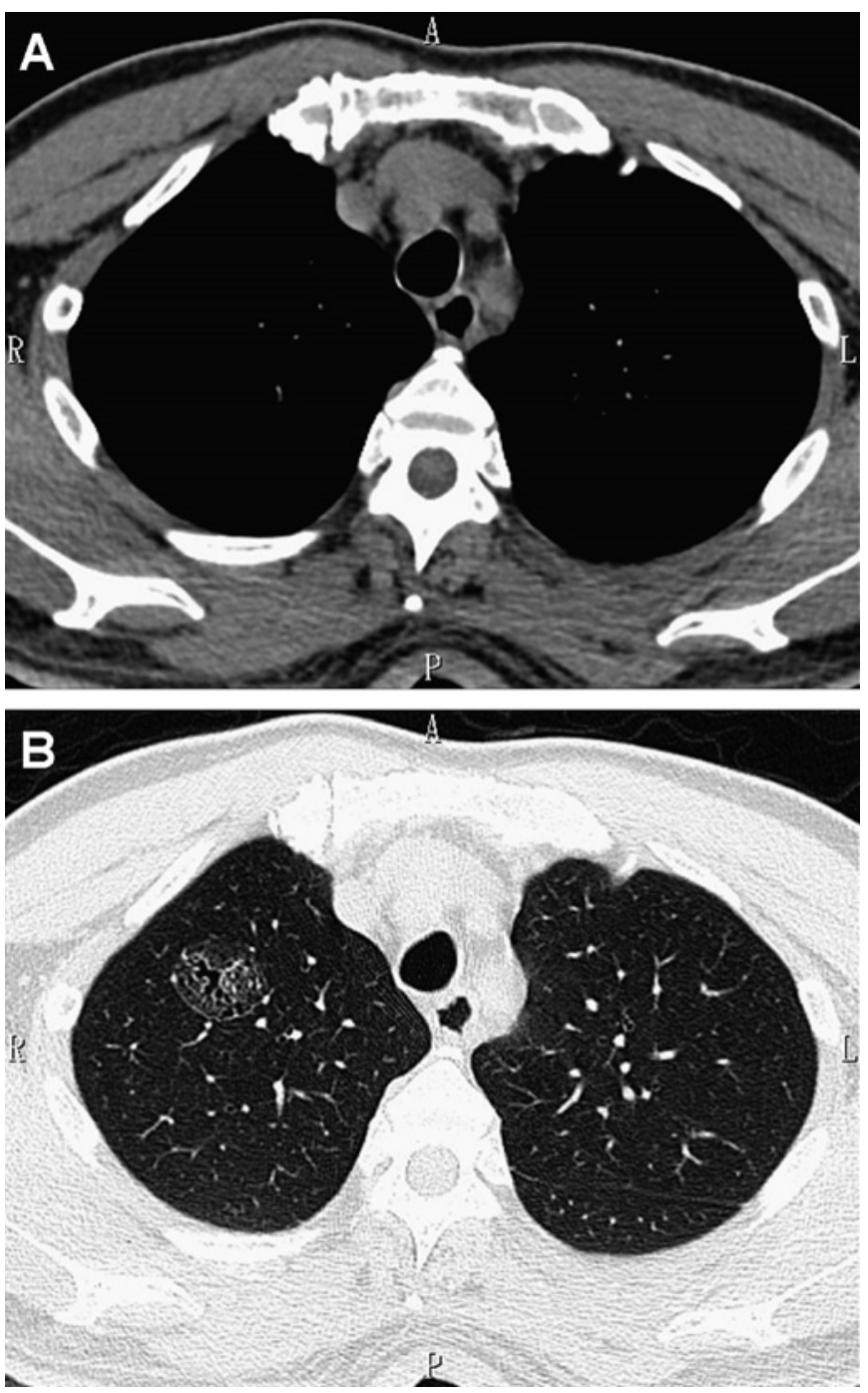

Figure 1 Chest CT image with soft tissue window settings $(A)$ and lung window settings $(B)$ showing a $2.8 \times 2.4 \mathrm{~cm}$ well-circumscribed, pure ground-glass opacity with air cyst formation in the right upper lobe. There was no identifiable soft-tissue component, calcification or fat within the lesion. tion to the nodule, a $2.8 \times 2.4 \mathrm{~cm}$ well-circumscribed, pure ground-glass opacity with air cyst formation was incidentally found (figure 1). There was no evidence of calcification or fat, and primary bronchoalveolar cell carcinoma was highly suspected. When performing preoperative CT-guided needle localisation of the opacity 2 weeks later, we found that the lesion remained unchanged. After video-assisted thoracoscopic wedge resection,
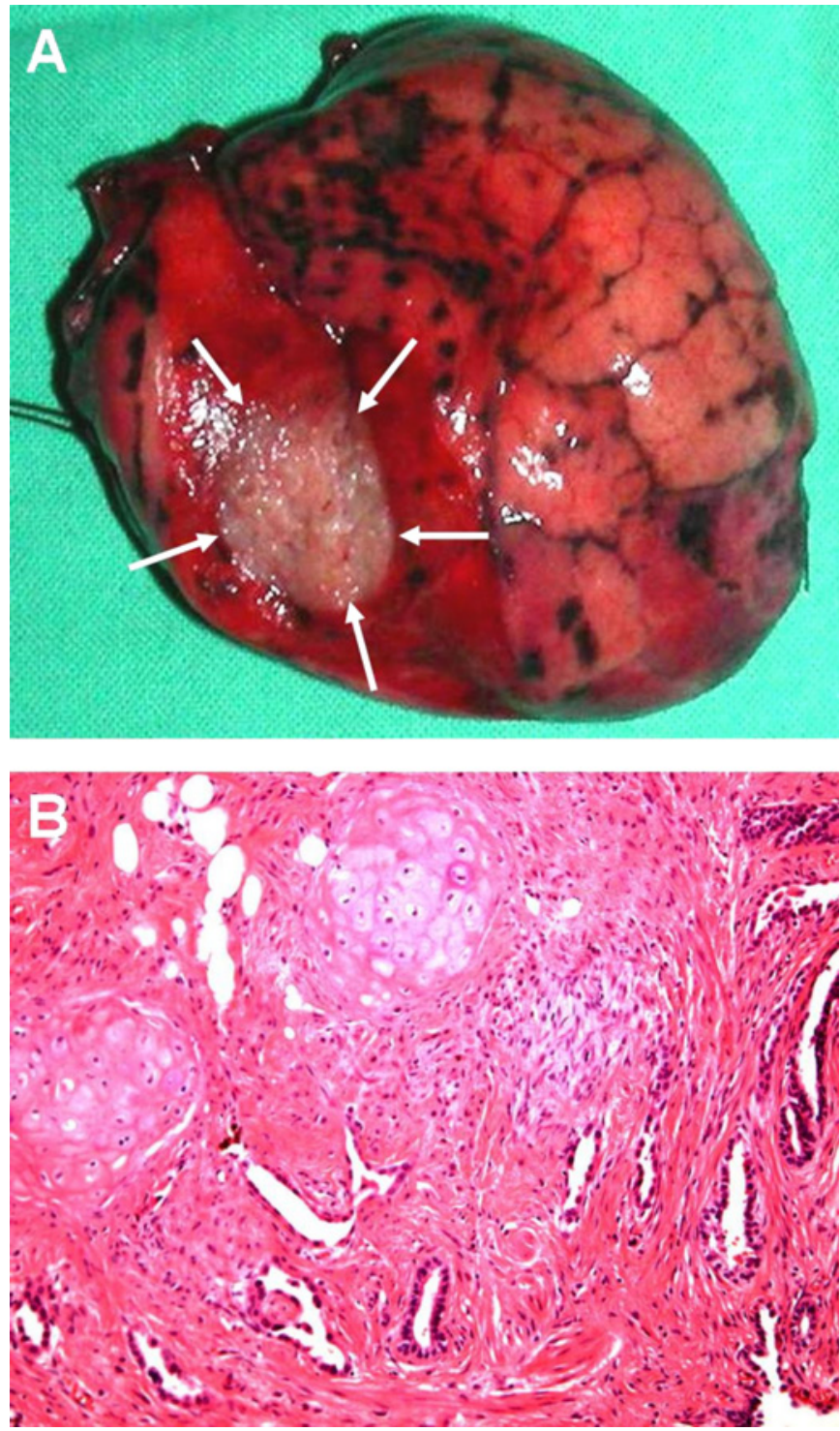

Figure 2 Gross morphology of the specimen showing a well-circumscribed, spongy and whitish tumour (arrows in A). (B) Pathologic examination demonstrating that the lesion was composed of cartilage, fat and smooth muscle with benign bronchial epithelial cells (H\&E staining $\times 100$ ). 